\title{
SISTEMA NORMATIVO, PRINCIPIOS IMPLÍCITOS Y FÓRMULA DEL PESO
}

NORMATIVE SYSTEM, IMPLICIT PRINCIPLES AND WEIGHT FORMULA

Sebastián de Jesús Chavarría Lara ${ }^{1}$

\section{RESUMEN:}

Se realizará una reconstrucción y análisis de la sentencia de la Corte Suprema de Justicia de Honduras, expedientes RI 1343-14 acumulada con el RI 0243-15. Para ello, se utilizarán las matrices lógicas para sistemas normativos de Alchourrón \& Bulygin, el modelo para inferir principios implícitos de Juan Pablo Alonso y el procedimiento de ponderación de principios de Robert Alexy.

PALABRAS CLAVE: Honduras, sistema normativo, normas, reglas, principios, Constitución, reelección presidencial

\section{ABSTRACT:}

This document presents a reconstruction and analysis of the judicial sentence of the Supreme Court of Justice of Honduras, file RI 1343-14 acumulated with RI 0243-15. For this, the logical matrices for normative systems of Alchourrón \& Bulygin are used, the model of inferring implicit principles of Juan Pablo Alonso and the procedure of principle weighting of Robert Alexy.

KEYWORDS: Honduras, normative system, norms, rules, principles, Constitution, presidential re-election

\section{Recibido: 29 de junio 2018}

Aceptado: 19 de septiembre 2018 


\section{INTRODUCCIÓN}

El propósito de esta investigación radica básicamente en establecer un punto de contacto entre los instrumentos conceptuales que producen los teóricos del derecho de distintas corrientes y cómo estos pueden ser útiles para describir y explicar lo que ocurre en la realidad. En este caso particular, nos interesa mostrar cómo las herramientas lógicas propuestas por autores como Alchourrón y Bulygin respecto de los sistemas normativos, así como la fórmula de la ponderación (de Robert Alexy), pueden servir para analizar sentencias.

Para lograr este cometido, hemos tomado como modelo para analizar, la sentencia proferida en abril del año 2015 según expediente RI 134314 acumulada con el Rl 0243-15 (en adelante la sentencia). En dicha sentencia se declaraban inaplicables los artículos que tratan la reelección presidencial en Honduras.

La sentencia es susceptible de diversos análisis, tal y como hasta ahora han propuesto muchos: dikelógicos², históricos, sociológicos, etc. Sin embargo, para lo que nos compete, estaremos presentado un análisis específicamente normológico ${ }^{3}$.

\section{METODOLOGÍA}

Para lograr nuestro análisis, haremos una reconstrucción de un sistema normativo, teniendo como base la concepción de las normas y de los sistemas normativos de Alchourrón y Bulygin (1998) y la distinción de MacCormick entre consistencia y coherencia.

2 Con dikelógico, en el sentido de qué tan apegado está al valor justicia; es decir, qué tan justo resulta que se haya llevado a cabo dicha decisión de declarar inaplicable la normativa constitucional.

3 Con normológico, hacemos referencia a esa parte de la fenomenología jurídica, que, al hacer un reduccionismo de la misma, se ocupa de las normas, su comportamiento y su relación con otras normas o principios. Tiene en cuenta, entre otros, instrumentos lógicos con los cuales puede hacerse un análisis del sistema normativo
La primera parte versará exclusivamente sobre el comportamiento de las normas declaradas inaplicables por inconstitucionalidad contenidas en la Constitución de la República; con ellas se reconstruirá un sistema normativo conformado por los artículos 42 numeral 5), 239 de la Constitución de la República de Honduras (CRH) y el 330 del Código Penal de Honduras (CPH) el cual fue declarado inconstitucional. La segunda parte del trabajo, tratará sobre la forma en que se contraponen los principios con las reglas. Para ello, se hará una abstracción de dichos principios y se utilizará la fórmula del peso tal como la plantea Alexy, para ponderar principios.

Para anteponer estos principios explícitos invocados por los impetrantes, tendrá que establecerse principios implícitos, los cuales puedan ser ponderados. Se utilizará el método inductivo para la obtención de principios implícitos, tal como lo propone Alonso. La sentencia no hace referencia alguna acerca de la metodología Alexyana para ponderar principios, sin embargo usa su lenguaje ${ }^{4}$, haciendo la mención de voces como ponderar, colisión, etc.

\section{Análisis y sistema normativo de la sentencia}

\subsection{Generalidades de la sentencia. Normas y principios implicados}

Haciendo una breve narración de lo contenido en la sentencia, podemos decir que la misma fue emitida en virtud de una interposición de acción de inconstitucionalidad, primero por parte de un grupo de diputados del Congreso Nacional de la República de Honduras, y después, también otra acción de inconstitucionalidad por parte del expresidente de la República, el Sr. Rafael Leonardo Callejas, quien

4 Puede verse en la pág. 4 de la sentencia: "relacionan las normas (las que fueron consideradas inaplicables) internacionales aprobadas por el Estado de Honduras, las cuales deberían de ponderarse (la negrita es mía), según la tesitura de los recurrentes por sobre los artículos constitucionales restrictivos de derechos que son objeto de la presente acción constitucional”. 
fue mandatario en el periodo 1990-1994. Al ser dirigidas contra el mismo acto, la Corte Suprema de Justicia de Honduras (en adelante CSJH) consideró procedente acumular los dos recursos de inconstitucionalidad registrados bajo los números 1343-2014 y $243-2015$.

Son muchos los artículos que se invocan en la sentencia, tanto por parte de los impetrantes como por parte de la misma Corte Suprema de Justicia. Sin embargo, hay tres artículos que son claves para el desarrollo de nuestro análisis. Estos artículos son: 42 numeral 5) y 239 de la $\mathrm{CRH}$ y 330 del $\mathrm{CPH}^{5}$.

Para facilitar la lectura y comprensión de los artículos acá tratados, a continuación, en el orden enunciados en el párrafo anterior los transcribiremos:

"Artículo 42: La calidad de ciudadano se
pierde:
1. $[\ldots]$
$2 . \quad[\ldots]$
$3 . \quad[\ldots]$
$4 . \quad[\ldots]$
5. Porincitar, promovero apoyarel continuismo
o la reelección del Presidente de la República."

"Artículo 239: El ciudadano que haya desempeñado la titularidad del Poder Ejecutivo no podrá ser Presidente o Designado.

El que quebrante esta disposición o proponga su reforma, así como aquellos que lo apoyen directa o indirectamente, cesarán de inmediato en el desempeño de sus respectivos cargos, y quedarán inhabilitados por (10) diez años para el ejercicio de toda función pública".

Estos artículos, son tomados de la Constitución de la República 6 .

5 Sentencia pág. 2

6 Constitución de la República Decreto No. 131 del 11 de enero de 1982, publicado en el Diario Oficial la Gaceta No. 23,612 del 20 de
A continuación, trascribo el artículo 330 del Código Penal hondureño:

"ARTICULO 330. Será sancionado con reclusión de seis a diez años quien habiendo ejercido a cualquier título la Presidencia de la República, promoviere o ejecutare actos violatorios del artículo constitucional que le prohíbe ejercer nuevamente la Presidencia de la República o desempeñar de nuevo dicho cargo bajo cualquier título.

En la misma pena incurrirán quienes 10 apoyaren directamente o propusieren reformar dicho artículo. Cuando los autores de esos delitos fueren funcionarios serán sancionados además con inhabilitación absoluta por diez años contados desde la fecha de la violación o de su intento de reforma".

Estos son los artículos que más nos interesan, pues son los que, en la resolución de la sentencia, se declararon inaplicables, los de la Constitución, e inconstitucional el 330 del Código Penal.

Luego tenemos los principios que se han aplicado para sustentar la resolución. Los diputados alegaron que con las disposiciones descritas arriba, tanto en la Constitución de la República como en el Código Penal, se conculca el principio de libertad de expresión y debido proceso ya que "al establecer penas proscriptivas, inhumanas y degradantes, como la destitución ipso facto de sus cargos sin debido proceso, así como la pérdida de la ciudadanía por el solo hecho de proponer dentro del marco legal un debate sobre los temas que la Constitución prohíbe y penaliza"'.

Por su parte, el expresidente Rafael Leonardo Callejas invocaba a los "derechos"8 a la igualdad como ciudadano, ser electo y la libre representación

enero de 1982. Tomado de la publicación elaborada al cuidado de OIM EDITORIAL S.A de C.V.

7 Sentencia pág. 3

$8 \mathrm{La}$ sentencia habla de derecho, en lugar de principios. Sin embargo, entenderemos que como derechos, se refiere a principios. 
ya que "le restringe participar libremente en condiciones de igualdad en sucesivas justas presidenciales en nuestro país y por estar en contradicción flagrante con normas constitucionales, de tratados y convenciones de los que Honduras es parte, misma que se integran de manera plena $y$ efectiva a nuestro derecho interno, como un todo único e indivisible"9.

La sentencia, sustenta la declaratoria de inconstitucionalidad de los artículos de la $\mathrm{CRH}$, en que estos restringen, disminuyen y tergiversan derechos y garantías fundamentales establecidas en la propia Constitución y en los Tratados sobre Derechos Humanos suscritos por Honduras antes de la entrada en vigencia de la Constitución de 1982. Por todo ello, se han inobservado los siguientes principios $^{10}$ :

- Legalidad

- Necesidad

- Igualdad; y

- Proporcionalidad

Se trata de los principios alegados por los impetrantes, añadiéndose el de necesidad y omitiéndose el de libertad de expresión.

Todo esto se puede ordenar mediante la siguiente tabla:

\begin{tabular}{|l|l|l|}
\hline \multicolumn{1}{|c|}{ Artículos } & $\begin{array}{l}\text { Principios en contra } \\
\text { (según impetrante) }\end{array}$ & $\begin{array}{l}\text { Principios en } \\
\text { contra (según } \\
\text { la CSJ) }\end{array}$ \\
\hline $\begin{array}{l}42 \text { numeral 5) } \\
(\mathrm{CRH}): \text { pérdida } \\
\text { de la ciudadanía } \\
\text { por promover } \\
\text { continuismo (re- } \\
\text { elección) }\end{array}$ & $\begin{array}{l}\text { Debido proceso (según di- } \\
\text { putados) }\end{array}$ & Necesidad \\
& & Proporcionaldad de expresión \\
\hline
\end{tabular}

9 Sentencia pág. 3

10 Sentencia, pág. 31

\begin{tabular}{|l|l|l|}
\hline \multicolumn{1}{|c|}{ Artículos } & $\begin{array}{l}\text { Principios en contra } \\
\text { (según impetrante) }\end{array}$ & $\begin{array}{l}\text { Principios en } \\
\text { contra (según } \\
\text { la CSJ) }\end{array}$ \\
\hline $\begin{array}{l}\text { 239 (CRH): refe- } \\
\text { rente a la reelec- } \\
\text { ción en sí }\end{array}$ & Igualdad como ciudadano, & Legalidad \\
Ser electo, y & Necesidad \\
La libre representación & Igualdad \\
\hline $\begin{array}{l}330 \text { (CPH): nor- } \\
\text { ma respecto al } \\
\text { delito por incum- } \\
\text { plimiento del } \\
\begin{array}{l}239 \text { y normas } \\
\text { relacionadas }\end{array}\end{array}$ & Libertad de expresión & Proporcionalidad \\
\hline
\end{tabular}

Nota: Tabla elaborada con ideas propias pero con información de la Constitución de la República de Honduras.

Es notable que en la sentencia la CSJ no explicite cuáles principios son incompatibles con el artículo 330 del CPH. Sin embargo, puede atenderse a los mismos principios con losque se declaran inaplicables el artículo 42 numeral 5) y el 239. Al respecto dice: "se declara la INAPLICABILIDAD DE LOS ARTÍCULOS 23 NUMERAL QUINTOY239 DE LACONSTITUCIÓN DE LA REPÚBLICA, por restringir, disminuir y tergiversar derechos y garantías fundamentales establecidos en la propia constitución de 1982, inobservado los principios de legalidad, necesidad, igualdad y proporcionalidad (la negrita es mía) que deben de imperar en toda sociedad democrática, según lo anteriormente expuesto y motivado"11.

\subsection{Conceptos bases para el análisis}

Para el desarrollo de esta obra, se realizarán las siguientes delimitaciones conceptuales.

Por norma se entenderá el sentido de uno o varios artículos de un cuerpo normativo-jurídico (o formulaciones normativas) ya interpretados. Para uso en las matrices de sistemas normativos, las formulaciones normativas deben interpretarse de tal forma que sean reformuladas en enunciados

11 Sentencia pág. 31 
moleculares y condicionales. El antecedente establece una clase de situaciones de hecho y el consecuente prescribe (obliga, prohíbe o permite) otra clase de acciones. Una norma como "si se da la clase de comportamientos $\mathrm{f}$, entonces es obligatorio hacer h" se simboliza Obh / f.

Por su parte, se entenderá que un conjunto de normas jurídicas puede reconstruirse como un sistema normativo. Un sistema normativo es un sistema deductivo axiomático en que al menos algunos de sus enunciados son normas. Un sistema deductivo axiomático es un sistema deductivo en que todos los enunciados del conjunto infieren de un sub-conjunto del sistema (llamado base axiomática). Finalmente, un sistema deductivo es un conjunto que contiene todas sus consecuencias lógicas.

La clase de situaciones en las que aparece el problema normativo constituye el Universo del Discurso (UD) de nuestro problema. La propiedad en común que comparten los integrantes del universo del discurso es la propiedad definitoria de este UD. La clase de las acciones sobre cuyo estatus deóntico nos preguntamos en un problema normativo, constituyen el Universo de Acciones (UA). Las propiedades que delimitan las circunstancias relevantes del UD para la solución del problema normativo, constituyen el Universo de Propiedades (UP). Toda propiedad del UP o un compuesto veritativo-funcional de propiedades, define un caso posible dentro del sistema. El conjunto de todos los casos, es el Universo de Casos (UA). El conjunto de todas las soluciones normativas previstas en las normas del sistema es el Universo de Soluciones o US $^{12}$.

Respecto a la consistencia y a la coherencia, nos atenemos, explicando las nociones de MacCormick, a las descripciones del iusfilósofo argentino Juan Pablo Alonso:

12 Todas estas definiciones son tomadas de un artículo en trabajo de Alejandro Guevara Arroyo, titulado Matrices lógicas para la reconstrucción de sistemas normativos estáticos, facilitado por el autor.
"La consistencia es una relación entre normas: un sistema normativo es consistente si carece de antinomias. La coherencia, por su parte, es una relación entre normas y principios: un sistema normativo es coherente con relación a un principio (o conjunto de principios) si sus normas se subsumen en tal principio (o conjunto de principio)" (Alonso, 2013, p. 358).

Dadas estas definiciones, es claro que "nada impide que un sistema normativo sea consistente (sin antinomias) pero incoherente con los principios que rigen en él" (MacCormick, como se citó en Alonso, 2013, p. 358).

En relación a la función descriptiva de la coherencia, Alonso (2013) señala lo siguiente: "la función descriptiva presupone que el sistema normativo es completo (carece de lagunas) y consistente (carece de antinomias). Además, es un sistema coherente ya que es posible describir su coherencia en función de los principios que se inducen de él" (p. 358).

En este trabajo, nos interesa sobre todo la función descriptiva, y de ella, la de inducir principios. Ello, para ponderar un principio implícito con respecto a uno explícito.

\section{Análisis del sistema normativo y principios}

\subsection{Matriz lógica del sistema normativo}

\subsubsection{Aclaratoria previa y conceptos básicos}

Antes de proceder a lo que acá compete, valdría hacer un análisis respecto de los artículos 42 numeral 5) y el 239 de la $\mathrm{CRH}$.

Para los efectos que acá nos corresponden, vamos a interpretar dichos artículos como reglas y no como principios. Esto, porque la forma en que están redactados, responden más a la fórmula deóntica del tipo $p \rightarrow O p(=P h \sim p=\sim P \sim p)$ (Bulygin, 1995; también Echave, Urquijo, Guibourg, 2008, p. 168). 


\subsubsection{Reconstrucción de Sistema Normativo.}

\subsubsection{1. Ámbito Fáctico}

El problema normativo que amerita solución mediante el sistema normativo, se podría plantear así: ¿En qué circunstancia el ciudadano que ha servido como Presidente de la República, puede asumir otro mandato para otro período de tiempo ${ }^{13}$

Se pueden considerar los siguientes universos de propiedades:

- OM: Obediencia del mandatario

Esto significaría que el mandatario asume las siguientes conductas: no busca reelegirse, ni reelegir.

- OT: Obediencia de terceros

Esto significaría en los casos en los que algún tercero (o terceros) asume la siguiente conducta: no busca reelegir.

Habría que especificar que con estas dos propiedades (OM y OT) queremos decir cualquier conducta que se interprete como aquella que busca la reelección. Dado que estamos en la reconstrucción de un sistema normativo formal, no nos detendremos en dar más especificaciones, pues de lo contrario, el universo de casos sería demasiado amplio y excedería el objetivo que nos hemos propuesto en este trabajo.

\subsubsection{2. Ámbito normativo}

El universo de soluciones es el siguiente:

- Opc: obligatorio pérdida de ciudadanía

- Oi: obligatorio inhabilitar

- Or: obligatorio reclusión

- Phs: prohibido sancionar

13 Como universo de discurso (UD).

\subsection{Formalización de los artículos:}

- Art. 42: Opc/ OM v OT

Traducido: Obligatorio la pérdida de ciudadanía al mandatario o tercero que no obedece

- Art. 239: P2: Oi/ OT v OM

"Obligatorio inhabilitar si el tercero o el mandatario no obedecieron".

Respecto al artículo 239 párrafo primero, no lo formalizamos ya que no es relevante para el universo de casos que aquí se plantean.

- Art. 330: P1: Or/ OM

"Obligatoria la reclusión para mandatario que no obedece".

P2: Or/ OT

"Obligatorio reclusión si tercero no obedece".

- Art. $69 \mathrm{CRH}^{14}$ : Phs/OM.OT

Estaríamos proponiendo un artículo más, además de los señalados en la sentencia, en virtud del artículo 69 de la misma Constitución que prescribe:

Artículo 69: la libertad personal es inviolable y sólo con arreglo a las leyes podrá ser restringida o suspendida temporalmente.

La cual podríamos traducir como esa imposibilidad del Estado para sancionar a aquellos que no estén dentro del universo de posibilidades en los cuales se pueda subsumir con arreglo a las leyes una restricción de la libertad.

Formalizado, tenemos como resultado lo propuesto en supra.

Esto, lo aclaramos, ya que no sería verdadero enunciar una laguna en caso de que tanto el Mandatorio Obedezca como que Terceros Obedezcan.

14 Constitución de la República de Honduras 


\subsubsection{Generación de matriz}

Para facilitar la lectura, a continuación presento las normas formalizadas en una sola columna:

N1. $\mathrm{Opc} / \sim \mathrm{OM} \vee \sim \mathrm{OT}$

N2. $\mathrm{Oi} / \sim \mathrm{OT} \vee \sim \mathrm{OM}$

N3. Or/ OM

N4. Or/ $\sim$ OT

N5. Phs/OM.OT

Por tanto, tenemos el siguiente Sistema Normativo:

$\mathrm{S} 1=\{\mathrm{N} 1, \mathrm{~N} 2, \mathrm{~N} 3, \mathrm{~N} 4, \mathrm{~N} 5\}$

Ahora, se presenta la relación del ámbito fáctico con el normativo respecto de las soluciones:

$\mathrm{N} 1: \mathrm{Opc} / \sim \mathrm{OM}$

\begin{tabular}{|c|c|c|c|}
\hline UA/UP & OM & OT & \\
\hline 1 & + & + & \\
\hline 2 & - & + & Opc \\
\hline 3 & + & - & Opc \\
\hline 4 & - & - & Opc \\
\hline
\end{tabular}

Sistema gráfico para observación de todo el comportamiento de las normas respecto del ámbito normativo:

\begin{tabular}{|l|l|l|l|l|l|l|l|}
\hline Sistema: & Casos & & Normas & & & & \\
\hline UA/UP & OM & OT & N1 & N2 & N3 & N4 & N5 \\
\hline 1 & + & + & & & & & Phs \\
\hline 2 & - & + & Opc & Oi & Or & & \\
\hline 3 & + & - & Opc & Oi & & Or & \\
\hline 4 & - & - & Opc & Oi & Or & Or & \\
\hline
\end{tabular}

N1. Opc/ $\sim \mathrm{OM} \mathrm{v} \sim \mathrm{OT}$

N2. $\mathrm{Oi} / \sim \mathrm{OT} \vee \sim \mathrm{OM}$

N3. $\mathrm{Or} / \sim \mathrm{OM}$

N4. Or/ O OT

N5. Phs/OM.OT

USmax: $\{$ Phs, Or, Oi, Opc $\}$

\subsubsection{Conclusiones respecto del sistema reconstruido}

De todo esto puede concluirse lo siguiente:

Tenemos un sistema cerrado y sin lagunas: para todos los casos, en cada caso hay una solución posible. No hay incoherencias, ya que si bien aparecen varias soluciones, no son incompatibles entre sí. Habría redundancia, pero no nos generaría problemas normativos importantes. Más bien, de ellos puede encontrarse indicios de principios implícitos.

Por tanto, a la pregunta formulada al inicio, ¿en qué circunstancia el ciudadano que ha servido como presidente de la república, tiene permitido asumir otro mandato para otro período de tiempo? Con contundencia se puede responder: en ninguna circunstancia. Siempre hay alguna sanción, llámese ésta reclusión ( $r$ ), inhabilitación (i), pérdida de ciudadanía (pc).

\subsection{Establecimiento de principios implícitos \\ 4.2.1.Los principios implícitos}

Para nuestro análisis, procederemos a detectar solamente un principio implícito, para, en un procedimiento posterior, someterlo a la fórmula del peso propuesto por el autor Robert Alexy. Así lo ponderaremos respecto a alguno de los principios explícitos enunciados en la sentencia. De esa manera, tendremos una herramienta de análisis comparativo.

Para inducir ${ }^{15}$ el principio implícito, se hará un

15 Sobre la inducción, nos atenemos a lo explicitado por Alonso: "En este contexto, por inducción me refiero al método clásico de las ciencias naturales, delineado por Bacon en Novum Organum (1620) y no a la <<inducción jurídica >>Alchourrón y Bulygin (1971, 130 y ss.), que es presentada como una forma de deducción al tratarse de inducción completa. Asimismo, podría utilizarse la abducción en lugar de la inducción, aunque con el mismo resultado, dado que ambas herramientas metodológicas llevarían a las mismas conclusiones, al menos el marco del presente trabajo. En otros contextos jurídicos, probablemente la abducción presente 
análisis de las relaciones lógicas de las normas de la Constitución ya revisadas en nuestra matriz (42 n. 5) y 239). No es necesario cotejar el 330 del $\mathrm{CPH}$ ya que el mismo quedaría dentro del principio lex superior de la Constitución sobre la ley. Pero dicha situación no se resolvería, según lo motivado por la CSJH, respecto de la Constitución que entendería la misma que se debe someter a "principios superiores sobre cualquier ley o incluso la Constitución”.

Los principios implícitos pueden entenderse como un mandato de optimización que, a diferencia de aquellos que son enunciados por una constitución o una ley, no están proclamados pero que pueden inferirse a partir de ciertas reglas. Esta cuestión lleva a plantearse lo siguiente: ¿Cómo explicar lo que es parte del derecho pero no está escrito? Al respecto puede verse el caso Riggs contra Palmer que es el fallo por referencia, en el cual se plantea esta posibilidad de que dentro de las mismas normas jurídicas (en tanto que reglas) estarían contenidos principios $^{16}$.

Respecto del principio implícito que tratamos determinar, proponemos que podría ser planteado de la siguiente manera:

"Quien no desobedezca la prohibición de la reelección está en mejor condición de conservar la ciudadanía, no ser castigado con inhabilitación y pena de reclusión"

Para darle más contexto a nuestra propuesta de principio implícito, se tomará en cuenta lo prescrito en el artículo 4 párrafos segundo y tercero de la $\mathrm{CRH}$.

ventajas metodológicas por sobre la inducción, en el sentido que sea la mejor explicación metodológica sobre el modo en que los juristas obtienen principios implícitos. Sobre la abducción en la ciencia jurídica puede consultarse Tuzet, 2010: 15-90" (Alonso, 2013, p. 358)

$16 \mathrm{Al}$ respecto, puede verse en la página web: http://www.rtfd.es/ numero11/21-11.pdf
"Artículo 4: [...]

La alternabilidad en el ejercicio de la Presidencia de la República es obligatoria.

La infracción de esta norma constituye delito de traición a la Patria".

Consideramos pertinente agregar este artículo en virtud de que la misma sentencia en sus fundamentos de derecho lo invoca ${ }^{17}$.

Continuando con la inducción de nuestro principio implícito, el mismo se referiría a una relación "mejor condición que..." que también puede interpretarse, para nuestro ejercicio, como "mejor derecho que". Por lo tanto, utilizaremos las herramientas de la lógica de relaciones, instrumento propuesto por Alonso (Alonso, 2013, p. 361 y ss) ${ }^{18}$.

Trataremos ahora de verificar la existencia de dicho principio

$$
\alpha: M O>\sim M O
$$

- 1 (MO\&OT) > 2 ( MO\&OT): bajo las condiciones constantes OT, MO (caso 1) tiene mejor derecho que $\sim \mathrm{MO}$ (caso 2): el caso 1 puede conservar la ciudadanía (Phs) mientras que el caso 2 debe perder la ciudadanía (Opc), inhabilitado (Oi) y bajo pena de reclusión (Or).

- 3 (MO\& OT) > 4 ( MO\& OT): bajo las condiciones constantes $\sim$ OT (caso 3 ), tiene mejor 17 Sentencia pág. 30

18 El método que propone Alonso, básicamente estriba en los siguientes lineamientos: en primer lugar se sigue la concepción de las normas tal y como lo proponen Alchourrón y Bulygin (esto está definido en supra). Y respecto a los principios implícitos, el tema importante es determinar un método que nos justifique ( ¿o $^{\circ}$ demuestre?) su existencia: en todo caso, debe ser posible que, de determinadas reglas, se pueda decir que esos principios existen. Para ello, se hace uso del método inductivo, en cuanto que se revisa el comportamiento de lo que las reglas ordenan (en tanto mandatos de todo o nada); se compara su reiteración y a partir de ella se induce el principio. Para ello, se los estructuran como fórmulas de la lógica de relaciones (Alonso, 2013, p. 358). 
derecho que OT (caso 3) -"no derecho"- que $\sim$ MO y OT (caso4): en ambos existe la obligación de que un sujeto pierda la ciudadanía, quedar bajo inhabilitación y bajo pena de reclusión, excepto MO (caso 3).

Con esto entonces quedaría verificado que en efecto hay un principio implícito que estaría bajo la relación "mejor condición que" de conservar la ciudadanía, no ser inhabilitado y no ser castigado bajo pena de reclusión; misma que podría entenderse como "mejor derecho que...". Esto, porque en efecto se prueba, a partir del Universo de Casos (UC) y las relaciones existentes, que $\mathrm{MO}>\sim \mathrm{MO}$.

Puede hacerse el mismo procedimiento con respecto a la relación b: OT $>\sim 0 T$, pero para nuestro ejercicio, no requerimos de otros principios implícitos para ponderar, ya que lo que tiene como objetivo este trabajo versa únicamente bajo la utilización de las herramientas propuestas por el positivismo en su parte de la lógica deóntica, lógica de relaciones y demás.

A esto, agregaremos lo antes mencionado respecto al artículo 4 párrafos segundo y tercero de la $\mathrm{CRH}$, que hacen referencia a la forma de gobierno, haciendo énfasis en la alternabilidad en el ejercicio de la Presidencia de la República ${ }^{19}$. Así entonces, para cerrar esta sección y tratando de sintetizar de una manera el principio inducido de la relación MO> MO lo catalogaremos como:

Principio implícito: obediencia a la institucionalidad constituyente.

Dado que el principio implícito tiene un nombre muy elocuente y con carga emotiva, preferimos en adelante, nombrarlo como principio $\alpha$.

Contando con el principio implícito $\alpha$, pasaremos

19 En un momento se trajo a discusión sobre los alcances interpretativos de la palabra "alternabilidad" ahora que la reelección es posible. Sin embargo, para el ejercicio del análisis de la sentencia, la alternabilidad implica la posibilidad de la reelección, pues, si la reelección era prohibida, también lo era la alternabilidad. Por tanto, no necesitamos considerar esa discusión. ahora a ponderarlo con alguno de los principios explícitos enunciados en la sentencia y veremos cuál sería el peso de cada uno de ellos.

\subsection{Análisis de los principios y aplicación de la fórmula del peso}

\subsubsection{La ponderación: aspectos generales}

Vamos a comenzar esta parte, recordando la ley de la ponderación: "cuanto mayor es el grado de la no satisfacción o de afectación de uno de los principios, tanto mayor debe ser la importancia de la satisfacción del otro" (Alexy, como se citó en Bernal Pulido, p. 2003) ${ }^{20}$.

Se partirá de la siguiente fórmula:

\section{GPi,jC = IPiC . GPiA . SPiC / WPjC . GPjA .} SPjC (Bernal Pulido, 2003, p. 5) $)^{21}$

\subsubsection{Nomenclatura ${ }^{22}$ :}

GPi,jC: Principio Pi en relación con el Pj circunstancial del caso concreto

\section{Pi: Principio i}

Pj: Principio j

20 A efecto de que la cuestión acá tratada se torne más compresible, precisamos lo siguiente: Entendemos por ponderación a: "la forma en que se aplican los principios jurídicos, es decir, las normas que tienen la estructura de mandatos de optimización. Estas normas no determinan exactamente lo que debe hacerse, sino que ordenan "que algo sea realizado en la mayor medida posible, dentro de las posibilidades jurídicas y reales existentes" (Bernal Pulido, 2003, p. 225)

21 Debido a la tipografía, la forma en que está expuesta la fórmula luce diferente a la del texto acá citado. No obstante, en su contenido, es la misma. Según Bernal Pulido, "Esta fórmula expresa que el peso del principio $\mathrm{Pi}$ en relación con el principio $\mathrm{Pj}$, en las circunstancias del caso concreto, resulta del cuociente entre el producto de la afectación del principio Pi en concreto, su peso abstracto y la seguridad de las premisas empíricas relativas a su afectación, por una parte, y el producto de la afectación del principio $\mathrm{Pj}$ en concreto, su peso abstracto y la seguridad de las premisas empíricas relativas a su afectación, por otra”. (Bernal Pulido, 2003, p. 229)

22 Para una mayor comprensión de lo acá planteado: ídem 
IPiC: Grado de afectación de Pi (peso concreto)

GPiA: Peso abstracto de Pi

SPiC: Seguridad de las premisas empíricas de Pi

WPjC: Importancia de satisfacción de Pj

GPJA: peso abstracto de $\mathrm{Pj}$

SPjC: seguridad de las premisas empíricas de Pj

\subsubsection{Enunciación de principios}

Vamos a ponderar entonces los siguientes principios:

a: nuestro principio implícito inducido de los artículos constitucionales en cuestión (42 numeral quinto y $239 \mathrm{CRH}$ )

Igualdad: la cual es planteada en la motivación de la CSJH. Escogemos este principio para nuestro ejercicio, ya que puede ir aparejada a la igualdad en general entre ciudadanos como igualdad para ser escogido y ser electo, pese a haber ejercido el cargo. Respecto a los impetrantes, según citado por la sentencia, se utiliza el concepto de igualdad acorde al artículo $61^{23}$ de la $\mathrm{CRH}$, pero lo expanden por lo prescrito en el Artículo $24^{24}$ de la Convención Americana sobre Derechos humanos, la cual, su redacción responde claramente a la de un principio.

Pi: igualdad

Pj: $\alpha$

\subsubsection{Asignación numérica}

4.3.4.1.Escala general para referir a la afectación de los principios:

\begin{tabular}{|c|c|c|}
\hline Leve: 1 & Media: 2 & Intensa: 4 \\
\hline
\end{tabular}

4.3.4.2. Asignación de los principios en la escala de afectación:

\begin{tabular}{|c|c|}
\hline Pi: 4 & Pj: 2 \\
\hline
\end{tabular}

4.3.4.3. Escala general para referir al peso abstracto:

Leve: 1

Media: 2

Intensa: 4

4.3.4.4.Asignación de los principios en la escala de peso abstracto:

\begin{tabular}{|c|c|}
\hline Pi: 4 & Pj: 2 \\
\hline
\end{tabular}

4.3.4.5. Escala general para referir a la seguridad de las premisas fácticas:

Seguro: 1

\begin{tabular}{|c|c|} 
Plausible: $1 / 2$ & $\begin{array}{c}\text { No evidentemente } \\
\text { falso: } 1 / 4\end{array}$ \\
\hline
\end{tabular}

4.3.4.6. Asignación de los principios en la seguridad de las premisas fácticas

\begin{tabular}{|c|c|}
\hline Pi: 1 & Pj: $1 / 2$ \\
\hline
\end{tabular}

\subsubsection{Aplicación de la fórmula}

Recogiendo todos los datos, explicitando los principios se observaría de esta forma:

\begin{tabular}{|c|c|c|c|}
\hline Principio & $\begin{array}{c}\text { Grado de } \\
\text { afectación }\end{array}$ & $\begin{array}{c}\text { Peso } \\
\text { abstracto }\end{array}$ & $\begin{array}{c}\text { Seguridad de } \\
\text { las premisas } \\
\text { empíricas }\end{array}$ \\
\hline Igualdad & 4 & 4 & 1 \\
\hline $\mathrm{A}$ & 2 & 2 & $1 / 2$ \\
\hline
\end{tabular}

23 Artículo 61: La constitución garantiza a los hondureños y extranjeros residentes en el país, el derecho a la inviolabilidad de la vida, a la seguridad individual, a la libertad, a la igualdad ante la ley y a la propiedad.

24 Artículo 24. Igualdad ante la Ley.- Todas las personas son iguales ante la ley. En consecuencia, tienen derecho, sin discriminación, a igual protección de la ley 
A continuación, procederemos entonces a la aplicación de la fórmula del peso

$$
\begin{aligned}
& \text { GPi, jC }=4 \cdot 4 \cdot 1 / 2 \cdot 2 \cdot 1 / 2=16 / 2=8 \\
& \text { GPj, iC }=2 \cdot 2 \cdot 1 / 2 / 4 \cdot 4 \cdot 1=2 / 16=0.125
\end{aligned}
$$

\subsection{Conclusiones respecto a la ponderación}

Puede concluirse que, según lo ponderado por la CSJH y expuesto en la motivación de la sentencia, el principio implícito a es completamente derrotado, pues al aplicarse la fórmula del peso, apenas representa 0.125 respecto del principio explícito igualdad, que arrojó la cifra de 8, por lo cual su satisfacción debe ser llevaba a cabo. De hecho, fue llevada a cabo, ya que por ello se declara la inaplicabilidad de los artículos 42 numeral quinto y 239 de la $\mathrm{CRH}$ así como la inconstitucionalidad del 330 del $\mathrm{CPH}$.

\section{CONCLUSIONES}

Primero, debemos resaltar que estos análisis han puesto de manifiesto y clarificado que en efecto los instrumentos lógicos resultan importantes para demostrar cómo ciertas tesis teóricas permean los razonamientos de los jueces. En este caso particular, los Magistrados de la Sala Constitucional de la Corte Suprema de Justicia de Honduras. Por otro lado, es de vital importancia que gracias a estas explicitaciones (respecto del sistema normativo reconstruido y de la ponderación puesta en relevancia), podemos afirmar lo siguiente:

a. Que la asignación de la cifra para ponderar al principio igualdad respecto del principio $\alpha$, son considerados respecto del razonamiento propio de la CSJH. Se trató de recoger la intencionalidad de parte de la CSJH. La cuestión, que podría plantearse del caso acá analizado, es hasta qué punto realmente esas cifras están legitimadas, pues, al no existir un peso abstracto al que pueda hacerse referencia de manera empírica, se podría hacer cualquier otra asignación dependiendo de la tendencia ideológica que asuman los jueces en cargo.

b. Un problema muy serio de la aplicación, es el de la baja intersubjetividad de este escenario, muy diferente a lo que sucede en otros casos, donde hay argumentaciones respecto de dos posiciones antepuestas en un juicio contencioso, en los que la parte podría presentar una valoración y la contraparte, otra. Sin embargo, no es este el caso, donde no existe anteposición ninguna en el escenario. Quienes estarían en desacuerdo, lo hacen de manera política y no en la evacuación del presente recurso de inconstitucionalidad dirimida en la sentencia.

c. Por otra parte, no aceptamos la crítica en que se ha hecho un reduccionismo de normas, es decir, que la ponderación sólo de un principio implícito con el de igualdad, no arroja una auténtica validación de la decisión de la CSJH. Por el contrario, la sujeción a las normas para la reconstrucción del sistema normativo y la derivación del principio implícito, se puede constatar como un intento de mantenerse todo en el rango mismo de lo planteado en la sentencia. Ciertamente, que, de haberse antepuesto dos partes en un proceso contencioso, donde se puede dar mayor argumentación (como hemos mencionado antes), la sujeción a las normas no serían un requisito necesario, ya que en lugar de presentarse un principio implícito, también podrían plantearse principios explícitos tales como los invocados tanto por los impetrantes como por la misma CSJH. A ello debemos agregar que esta objeción no es correcta, ya que no existe hasta ahora una herramienta conceptual que haga un "cálculo de principios", con una fórmula tal como: "la mejor solución de todas, es aquella que satisface la mayor cantidad de principios" (como señaló Guibourg, 2011). Por lo tanto, 
consideramos que la aplicación de la fórmula del peso -tal y como la hemos propuestoal menos ilumina objetivamente cuál fue la relación entre los principios que se dio en el razonamiento de la CSJH para dar a lugar a la petición de los impetrantes.

d. Es necesario resaltar eso: la relación entre principios. La CSJH directamente ponderó las normas respecto de los principios invocados.

e. Finalmente, otra cuestión interesante que sobresale en este análisis, es la relación entre el principio implícito y los principios explícitos. Hasta ahora, no conocemos que se haya hecho una ponderación entre un principio implícito contra un principio explícito. No vemos que esto sea problema ya que al fin, la estructura del principio implícito responde también a la manera de un "mandato de optimización" que sólo en la estructura de la norma tiene una mayor precisión.

No aceptaríamos tampoco la objeción de que ese principio implícito no pueda ser ponderado respecto a un principio explícito, ya que, al ser inducido el principio implícito de una norma Constitucional, éste, por tanto, tiene dicho rango.

También, pensamos que quedan abiertas muchas otras preguntas, sobre todo en el sentido de cómo se puede dar más profundidad en la relación entre los principios implícitos y explícitos.

\section{BIBLIOGRAFÍA}

- Alchourrón y Bulygin, (1998). Introducción a la metodología de las ciencias jurídicas y sociales. Buenos Aires: Astrea.

- Alexy, Robert (2014). Principios formales. DOXA, Cuadernos de Filosofía del Derecho, núm. 37, pp. 15-29
- Alonso, J. P, (2013). Principios Jurídicos Implícitos y Coherencia. DOXA, Cuadernos de Filosofía del Derecho, num. 36, 357385.

- Bernal Pulido, Carlos (2003). Estructura y límites de la ponderación. DOXA, Cuadernos de Filosofía del Derecho, núm. 26, pp. 225-238

- Bulygin, E. (1995). “Lógica deóntica”. En: C. E. Alchourrón, J. M. Méndez Rodríguez, R. Orayen, Lógica (11-48). Madrid: Trotta.

- Echave, D.T., Urquijo, M. E., Guibourg, R. A. Lógica, proposición y norma. Editorial Astrea. Colección Filosofía y derecho vol. 9. 2008. P.168

- Guevara Arroyo, A. Matrices lógicas para la reconstrucción de sistemas normativos estáticos. Documento en preparación facilitado por el autor.

- Guibourg, R. (2011). "Alexy y su fórmula de peso". En: Desafíos de la ponderación (eds. G. Beade \& L. Clérigo), 157-187. Bogotá: Universidad Externado de Colombia. 